\title{
Evolution of mechanoregulation of bone growth will lead to non-optimal bone phenotypes
}

\author{
NIAMH C. NOWLAN and PATRICK J. PRENDERGAST* \\ Centre for Bioengineering, \\ Department of Mechanical Engineering, \\ Trinity College, \\ Dublin, \\ Ireland
}

*Corresponding Author. Tel: +353-1-608-1383; fax +353-1-679-5554.

E-mail addresses: pprender@tcd.ie (P. J. Prendergast), nowlann@tcd.ie (N.C. Nowlan). 


\begin{abstract}
Mechanical forces acting on the bones during growth affect their final shape and strength. Mechanoregulation of bone growth may be recognised in embryogenesis, and also in the adaptation of the adult skeleton to changes in mechanical loading. Mechanoregulatory responses for tissues have arisen during evolution, but does evolution give rise to responses that produce optimal skeletal phenotypes? In this paper, we investigate the emergence of an optimal mechanoregulation response in a population.

By combining equations describing long bone growth with a genetic algorithm to describe evolutionary change, we created a computational model to simulate the evolution of mechanoregulation in bone growth. A population of individuals is created where each individual is assigned a diploid gene set which controls the growth and remodelling of the bone. At maturity, each bone is assessed and its 'fitness' calculated; fitness is quantified as bone strength relative to bone mass. The simulation continues for many generations, and includes mutations and a varying environment. The genes present in the population are tracked and the evolution of parameters governing mechanoregulation is calculated.

The results indicate that a population may converge to one bone growth algorithm but, more usually, a range of mechanoregulation algorithms for different individuals will persist after many generations. Even if the population converges to one mechanoregulation law, convergence to the 'optimum' bone was never found. Although many researchers propose that natural selection has pushed skeletal structure towards an optimum, our computational model suggests that this is unlikely to be the case.
\end{abstract}

Keywords: Mechanoregulation algorithms; Bone growth; Optimality; Modelling evolution; Genetic algorithm. 


\section{Introduction}

The development, growth, and remodelling of skeletal structures is a highly regulated process beginning with mesenchymal stem cell condensations in the early embryo and finishing with the homeostatic skeleton of the adult. It is widely accepted that both genetic and epigenetic factors determine the final shape and strength of the skeleton, and many authors have specifically proposed an epigenetic role for mechanical forces (Pauwels, 1941; Thompson, 1917; Wolff, 1892). Equations have been proposed to describe how mechanical forces modulate growth where a mechano-biological growth rate is superimposed on a baseline biological growth rate (Carter and Beaupré, 2001). Since the final skeletal structure is partly determined by mechanobiological growth, the mechanoregulatory responses that produce fitter skeletal structures should come to the fore in a population (Prendergast, 2002). Alexander (1981) investigated the most economical possible design for a bone of specified strength, and found that there exists an optimum ratio of inner to outer bone radii for strength with lightness. Currey and Alexander (1985) propose similar optimal ratios, assuming that the bones are designed to have a minimum mass to perform a particular function. At present, simulations of the growth and adaptation of skeletal structures are based on deterministic growth laws where the possible variation in the population is not included (van der Meulen and Huiskes, 2002).

Biomechanical modelling of bone growth and adaptation was presented by Cowin and Hegedus (1976) who proposed an open system in which the rate of change of bone mass was related to mechanical strain. Skalak et al. (1982) later formulated a continuum model of growth. Carter et al. (1987) proposed bone remodelling was targeted to produce a homeostatic level of an 'effective stimulus' and Huiskes et al. (1987) proposed that the process of bone remodelling was an adaptation that returned the strain energy density in the tissue to a homeostatic value. Bone growth models incorporating both biological and mechanobiological influences have been proposed by van der Meulen et al. (1993), for modelling the cross-sectional growth of long bones, 
and by Stevens et al. (1999), for modelling endochondral growth using a finite element model. An alternative approach is that remodelling of bone is in response to microdamage (Martin, 2000), either to regulate microdamage to a homeostatic level (Prendergast and Taylor, 1994) or as the stimulus for the activation of the coupled responses of osteoclast and osteoblast cells (Huiskes et al., 2000). Recently, the molecular genetics of bone growth and development has received much attention to identify, for example, the genes that influence bone mineral content (Karasik et al., 2002), bone geometry (Klein et al., 2002; Turner et al., 2004), or lead to bone disorders such as osteoporosis (Peacock, 2002), and osteopetrosis (de Vernejoul and Benichou, 2001). Experiments with embryos have established the importance of genes such as Indian hedgehog (Long et al., 2004; Vortkamp et al., 1996), parathyroid hormone-related protein (Lanske et al., 1996) and fibroblast growth factors (Deleziode et al., 1998; Deng et al., 1996) for bone growth and development. While the size and mineral content of a bone are influenced by the processes of bone remodelling, it is probable that genes exist with the sole function of controlling bone response to mechanical loading, (i.e. mechanosensitive genes, Carter et al., 1998). Despite recent advances in the molecular biology of bone, the heritability and variance of the genes controlling bone growth and development remain as yet unknown (Boyce at al., 1999).

We propose to create a model to simulate how mechanoregulation of bone growth is subject to selection pressures during evolution. To do this a genetic algorithm is used which relates the differential equation describing growth to a simplified representation of the genome. The simulation should give outputs describing the population genetics of bone growth and remodelling, i.e. the variance present in a population, and how or why this variation arose, or is maintained, through processes such as recombination through reproduction, changes in the external environment (de Jong and Gavrilets, 2000), genetic mutations (Waxman and Peck, 2003), and migration and random drift (Marroig and Cheverud, 2004; Tufto, 2000). The specific aim is to investigate whether or not changes in mechanoregulatory response can occur during evolution and, if so, how would this help us to better represent the adaptive behaviour in the 
individual skeleton. In particular, will all individuals in a modelled population reach a biomechanical optimum?

\section{Methods}

\subsection{Model genome and its relationship to the growth law}

Computational simulations are used to investigate the mechanoregulation of bone growth and development in a population over many generations. The bone growth model employed was proposed by van der Meulen et al. (1993). It simulates the growth of the cross section of a long bone from an embryonic bone collar to maturity, where the rate of bone apposition or deposition If is equal to the sum of the baseline biological rate $\&$ and the rate due to mechanobiological effects $\&_{m}^{\&}$ as defined in equation 1 .

$\stackrel{\&}{\&}=k_{b}^{\&}+i_{m}^{\&}$

The baseline biological rate is a decaying exponential function of time that decays to approximately zero by six years, as defined in Eqn. 2 .

$\mathbb{L}_{b}=\beta e^{-0.9 t}$

where ${ }_{\sigma} \&$ is the baseline rate magnitude and $\beta$ is the initial rate magnitude, taken as $10 \mu \mathrm{m} /$ day in this model. The rate due to mechanobiological effects is dependant on the daily stress stimulus $\psi$, where the rate of change of radius due to mechanobiological effects is defined in Eqn. 3.

$\underset{m}{\&}=c\left(\psi-\psi_{A S}\right)$

where $\&_{m}$ is the rate of bone apposition or resorption on either the inside (endosteal) or outside (periosteal) surfaces of the bone due to mechanobiological effects, $\psi$ is the daily stress stimulus, $\psi_{\mathrm{AS}}$ is the attractor state (or desired level) stimulus on the bone, and $c$ is a growth rate constant. The daily stress stimulus $(\psi)$ denotes the mechanical stimulus to which the bone is exposed, and is calculated based on the number of cycles of a load, the tissue level effective stress and an empirical stress exponent (van der Meulen et al., 1993). The constant $\mathrm{c}$ is in fact a time constant, 
which will affect how quickly the bone reacts (via apposition or resorption) to the stresses placed upon it. A higher value of $\mathrm{c}$ will lead to more expansion and thus a thinner bone. Through the rate constant $c$, the variation and convergence of the population is assessed.

We propose that the magnitude of $\mathrm{c}$ is determined as follows: a diploid gene set is defined for each individual, taking a finite number of loci, in this case five as shown in Fig. 1. A simulation commences with the selection of 50 random numbers to form the gene pool; no new genes can be created except by mutation. The genes making up the gene set are numbers in the interval 0 to 1 , although genes greater than 1.0 may result from mutations. Each locus is filled with a gene selected at random from the subsection of the gene pool allocated to that locus (i.e. the gene pool is divided into 5 sets of 10 genes, one set per locus). The $c$ value is determined by the sum of the average of the genes at the 5 loci of gene sets $a$ and $b$;

$$
c=\sum_{i=1}^{n}\left(\frac{g_{i a}+g_{i b}}{2}\right),
$$

where $n$ is the number of genes, and $g_{i a}$ and $g_{i b}$ are genes from the a and $b$ gene sets at locus $i$ (as shown in Fig. 1). The genes have no dominance, therefore the effect of having genes g1g2 is midway between the effects of having genes g1g1 and genes g2g2 (Hartl and Clark, 1997), i.e. co-dominance is assumed.

Using Eqn. (3) and Eqn. (4), a mechanobiological growth law is derived from the diploid gene set. Using this law, the bone of each member of the population is grown as described below. The fitness of each individual is assessed when they reach maturity, taken to be 20 years in this model. For the bone of a mammal, it is important to minimise mass, as heavy bones are more expensive to maintain. It is also important to minimise the maximum stress the bone is subjected to (in order to avoid breakage of the bone), which will be the periosteal stress, assuming the bone is in torsion. We use stress/mass as a measure of fitness, such that the bone with the minimum value of $\tau_{\max } /$ mass will be at an optimum. The lower the maximum stress on the bone, the less likely it is to fail. Therefore, the lower the $\tau_{\max } /$ mass, the fitter the bone. Individuals are selected 
for recombination according to their fitness relative to the rest of the population. For a population

of $p$ individuals, a number $x$ is calculated such that $x=\sum_{i=1}^{p} i$, and the population is arranged in an ordered list such that the least fit individual is first and the most fit last. If $j$ is the position in the list, each individual is assigned a probability of selection of $j / x$. The probabilities of selection for all individuals sum to one, and a number line is divided up in proportion to these probabilities, where the least fit individual will occupy a region of size $1 / x$ on the number line, and the most fit will occupy a region of size $p / x$. A random number between 0 and 1 is obtained, and the individual located at the space on the number line corresponding to this random number is selected for recombination. In this way, fitter individuals are favoured, while unfit individuals still have a chance of being chosen for recombination, and an element of randomness is still maintained in the selection process.

Once an individual has been selected for recombination, a 'germ' or haploid gene set is obtained from the genes of the 'parent'. The genes in the haploid germ are taken at random from the parent. Two parents are selected to form one child, as shown in Fig. 2.

\subsection{Mutations}

Mutations take the form of randomly halving or doubling the value of the gene to be mutated. Each gene has a $10^{-5}$ chance of being mutated (Roberts and Pembrey, 1985), and the mutations take place after the creation of a new gene set.

\subsection{Epigenetic variation}

The van der Meulen et al. (1993) model uses an age-dependant loading moment to simulate the loading history for an individual bone as shown in the mean curve in Fig. 3. This moment was obtained from human body weight during growth (McCammon, 1970), and it was assumed that torsional moments and body weight would scale similarly with age (van der Meulen et al., 1993), 
to give a plot of age versus applied torsional moment. Differences between individuals' nutrition, climate and exercise levels will result in a range of loading moments, and to account for this, an envelope of $\pm 10 \%$ was plotted about the mean curve, as shown in Fig. 3. Each individual is assigned a moment-age curve at random from within this envelope.

\subsection{Varying selection strength}

Changes in lifestyle (Ruff et al., 1984) or changes in the environment (Raup, 1986) can cause the demands and/or fitness criteria on a bone to change. We include this in our model, where the effect of shifts in the environmental conditions can be investigated by varying the strength of selection cyclically. A high value for the strength of selection results in a competitive environment in which a high fitness conveys a significant reproductive advantage, whereas a low selection strength results in a more equal environment, where having an efficient bone conveys a less significant advantage. In the simulations where a variable selection force is used, the strength of selection is varied cyclically between 1.0 and 0.5 , where the value is changed by 0.1 every ten generations.

\subsection{Implementation}

A gene pool is created and a population of $n=1000$ individuals is initialised by assigning each individual a gene set. An environment is created, where the initial strength of the natural selection force is specified. In the next stage, mechanobiological laws are applied to grow each bone in the population to maturity. An individual's growth is determined by their gene-set and by the loading conditions applied. Parents are determined based on the relative fitness, or viability, of the individuals in the population, and the selection of parents is influenced by the current strength of the selection force, which can vary over time. Once a new generation has been created using the genes of the parent generation, the old generation is removed from the simulation. The passage of genes can be observed and analysed through generations. The simulation is run for 1,000 
generations, equivalent to 20,000 years, as each generation is assessed and culled at 20 years of age. One iteration entails the growth, reproduction and death of one generation. The steps involved in one iteration are illustrated in Fig. 4. The model is implemented in Java ${ }^{\mathrm{TM}}$, http://java.sun.com, using techniques adapted from genetic algorithms, as described by Holland (1975).

\section{Results}

\subsection{Behaviour of the bone growth model}

The magnitude of the growth rate constant $c$, as given in Eqn. (4) affects the final shape of the bone at 20 years, where higher values of $c$ result in a bone with greater periosteal and endosteal radii and correspondingly thinner cortical thickness. The constant $c$ is a time constant, and the higher the $c$ value, the quicker the bone will react to stresses by increasing its radii.

Assuming that every bone is grown with the same mean moment curve (Fig. 3), an optimal bone, (i.e., the lowest $\tau_{\max } /$ mass value at maturity) is created if $c=1.29$, see Fig. 6 . Therefore, under average loading conditions a mechanoregulation rule with $c=1.29$ will give the highest probability of survival. However, the loading conditions applied to the growing bone will affect its growth and resultant shape. We found that applying a lower torsional moment results in increased fitness with increasing $c$, and applying a higher moment leads to a decreased fitness with increasing $c$, as shown in Fig. 7.

\subsection{Behaviour of the evolution simulation}

Four kinds of simulation were performed:

Test 1) basic simulation,

Test 2) simulation including mutations,

Test 3) simulation including varying selection strength,

Test 4) simulation including both varying selection strength and mutations. 
Each test was repeated twice (Run A and Run B) with the same initial gene pool and population configuration; the two runs could be expected to give different results because of the randomness of recombination. This duplication enabled us to analyse the effects of the processes occurring within the simulation, independently of the initial conditions. Therefore each series had eight simulations consisting of two runs (Runs A and B) of each of the four tests listed above, and the complete series was repeated four times for four different initial gene pools (Gene-pools 1-4). Therefore 32 simulations were performed altogether.

The evolution of the gene values and the corresponding $c$ values were recorded for each individual throughout the simulation. These parameters can be used to compute variance (or lack thereof) in the population as evolution progresses. There are two possible final outcomes for each test: convergence to a single value of $c$, or non-convergence where $c$ is multi-valued in the population and there are many genes. Therefore convergence implies that every individual in the population has the same gene set after 1000 generations whereas non-convergence implies that variance is present in the population. If the population converges, only 5 genes will be left in the gene pool, i.e. each of the 5 genes occupies the same locus for every gene set, where the gene is replicated twice. In the case of non-convergence, more than 5 genes will be present in the gene pool, and a range of $c$ values will exist. It was found that some simulations converged whereas others did not; furthermore convergence did not occur to the same $c$ value, nor did convergence occur to the optimal value of $c=1.29$, see Table 2 .

\subsubsection{Detailed test results}

\section{Test 1: Basic simulation}

A basic simulation involves initialising a population and running the model without any genetic mutations or changes in the strength of the selection force. A basic simulation does however, like all of the simulations, include the epigenetic variation in applied loading. The initial population configuration has a large impact on determining the outcome of this particular simulation, 
because when two simulations were run with identical initial population configuration, the simulations could either both converge or both maintain variation, i.e. non-convergence occurs for both Run A and Run B in Gene-pool 1 whereas convergence occurs for both Runs A and B in Gene-pools 2, 3, and 4. In Run A of Gene-pool 1, a total of 13 genes still remain in the gene pool after 1000 generations, see Fig. 8 (a), giving rise to a range of $c$ values from $c=1.105$ to $c=$ 1.585 (Fig. 8(b)). It is important to note that in those cases where convergence occurs in both runs $\mathrm{A}$ and $\mathrm{B}$, the populations do not converge to the same $c$ value. For example, for the populations in Gene-pool 2, Run A converges to $c=1.415$ whereas Run B converges to 1.39. Although the populations have both converged, they have different final gene pool configurations, which give rise to different $c$ values. Fig. 9(a) shows the gene structure and Fig. 9(b) shows the $c$ value for one of these converged simulations, i.e. Gene-pool 2, Run A.

\section{Test 2: Simulation including mutations}

This test includes random mutations at the rate of $10^{-5}$ per gene per recombination. It was found that variation often remains in a population, but also that convergence can sometimes occur. When we ran two tests with the same initial population in Gene-pool 3, we saw that the population for Run A converged whereas the population in Run B maintained variance after 1000 generations. Therefore, unlike the situation with the basic simulation alone, the initial population configuration does not determine whether a population will converge or not. This means that the random processes contained within the simulation have a significant impact on the outcome. However, one feature remains the same as the basic simulation: if two tests within the same Gene-pool converge (e.g. Gene-pools 1, 2 and 4), then they converge to different $c$ values (see Table 2). 


\section{Test 3: Simulation including varying selection strength}

In this test, the strength of the selection force is varied cyclically throughout the course of the simulation. Where two tests were performed with the same initial population configuration, one of the tests converged (Gene-pools 1 and 2), while the other maintained variation. Therefore, we found that the population can either converge or maintain variation after 1000 generations and, similar to the Mutations test, the outcome is not determined by the initial population configuration. In Gene-pool 3, the populations converged to different $c$ values, while in Genepool 4, variation was found in both populations after 1000 generations. However, a different gene pool was maintained by the populations in Runs A and B in Gene-pool 4, and therefore a different spread in $c$ values.

\section{Test 4: Simulation including varying selection strength and mutations}

The fourth and final type of test includes both varying selection strength and mutations. Once again, we found that if two tests are run with the same initial population configuration, the population can either converge or maintain variation in the gene pool; this occurred in Gene-pools 2 and 3 (Table 2). In Gene-pool 1, both populations maintained variation, while in Gene-pool 4 , both populations converged, but to different values of $c$. Looking closer at the nature of this difference, Gene-pool 2, Run A converged to a gene pool of only 5 genes, which gave rise to a single $c$ value of 1.115 (Fig 10(a) and Fig. 10(b)). However, Gene-pool 2, Run B maintained many genes (Fig. 11(a)) giving rise to a wide distribution of bone growth rate constants (Fig.11 (b)).

\subsubsection{Gene Frequency Data}

The passage of each gene in the gene pool may be tracked throughout the simulation to obtain gene frequency graphs. Since there are 1000 individuals in the population, and each individual has 10 genes ( 5 diploid loci), each of the 5 genes will have a frequency of 2000 when dominant in 
the population. If a population converges, it is possible to see that most of the genes gradually decrease in frequency to zero, while 5 genes rise slowly to total dominance in the population. For Gene-pool 2, Run A, as previously described, convergence to a single $c$ value occurs. This convergence is reflected in the gene frequency data, where 5 genes can be seen rising in frequency until they reach dominance at 850 generations (Fig. 12). Gene-pool 2, Run B maintained variance in the population after 1000 generations and this can be seen in the gene frequency data, where a large number of genes are still present in the gene pool after 1000 generations and only 2 genes are approaching dominance (Fig. 13).

\section{Discussion}

The results obtained suggest that the processes of natural selection will not optimize the mechanoregulation of growth within a population. Therefore a biomechanically optimal bone will not be grown. The bone growth rate constants listed in Table 2 show that many of the populations converged to a $c$ value far from the optimum value of 1.29 ; therefore, this model would suggest that natural selection does not even guarantee that mechanoregulation will converge so that bones will grow to some near-optimal state. Another interesting aspect to the results is that the populations often do not converge to one single value for $c$, but instead maintain a range of values after 1000 generations. Therefore, if the model is accepted, these results demonstrate that variance can exist among the parameters governing mechanoregulation during growth. In the four types of tests we carried out, there was no test that consistently either converged or maintained variation, and so it is impossible to predict the result until a test is run. For the basic simulation (no mutations and no shifts in external environment), we found that the initial population configuration has a significant impact on the final result, as simulations run with the same initial population gave the same result, i.e., either convergence or maintenance of variation. However, although they gave the same outcome in respect of convergence vs. non-convergence, they did 
not yield the same final gene pool, and therefore the resultant populations were still different. For the other three tests (mutations, varying selection strength, varying selection strength including mutations), we found that the random evolutionary processes determine the results because, even when two tests of the same type were run with the same initial population configuration, the tests often displayed different final results, with one test converging and the other maintaining variation. In addition, if two tests of the same type and initial configuration both converged, they would converge to different $c$ values, giving, in effect, mechanoregulation algorithms for bone growth.

As with any model, a number of necessary simplifications have been included. Firstly, the bone growth model is rudimentary in that only cross-sectional growth of circular sections is considered. Secondly, the fitness of the bones is judged based on $\tau_{\max } /$ mass, where the stress is calculated based on the maximum torsional moment applied. If other loads, such as axial or compressive loads were also included, it is likely that a different bone shape would be optimal, and therefore there would be a different optimal value of $c$. Currey and Alexander (1985) examined several different modes of failure for long bones (such as buckling, fracture etc.), and discovered that different theoretical optimal bone shapes exist for different failure modes. However, introducing a more complex loading would not change the effects of the processes contained within the model, nor the nature of the results obtained. Thirdly, with a computational model, it is impossible to replicate the complexity of the human genome, and our representation of the genes as numbers having linear effect is obviously simplistic. Also, it is possible that a gene set of only five diploid genes is too limited, and that a larger gene set would allow more recombinations and mutations to occur and therefore individuals might have a better chance of reaching an optimum. However, our knowledge of the genes that impact on bone growth and remodelling, although much advanced, is still incomplete (Boyce et al., 1999) and it is not known yet exactly how many genes impact on bone growth and remodelling (Gilbert, 2000). Finally, not every detail of population genetics has been included in the model; for example, epistemic 
interaction between the loci of the gene sets, or dominance of certain genes. Nonetheless, as it stands, the genetic part of the model includes many realistic features, such as a diploid recombination, co-dominance of alleles, frequencies of mutations, and interaction between the genotype and the environment. A varying environment is also representative of the real situation.

Although some aspects of the model are simplistic, it does represent the main features of molecular genetics relevant to the question posed in the introduction. For example,

(i) Genes for recombination are selected at random from the parent chromosomes. This occurs if the genes in question are not on the same chromosome, but located on several different chromosomes, which has been shown to be the case for the genes that influence bone mineral density (BMD) in humans (Karasik et al., 2002), and bone geometry in mice (Klein et al., 2002; Turner et al., 2004). It is therefore acceptable to assume that the genes influencing bone growth are on different chromosomes, and this assumption is reflected in the model.

(ii) We use a mutation rate of $10^{-5}$ per gene, which corresponds to the rates of between $10^{-5}$ and $10^{-6}$ new mutations per gene per recombination suggested by Roberts and Pembrey (1985).

(iii) $\mathrm{A} \pm 10 \%$ variation in applied torsional moment is applied to the population. In the age vs. weight data of McCammon (1970), a standard deviation of roughly $10 \%$ is present for many of the early years, which shows that $\pm 10 \%$ is a good approximation for loading variability, as the loading is assumed to scale to weight vs. age data (van der Meulen et al., 1993). This variation represents the influence that environmental variation can have on the growth of a bone, such as the studies effects of nutrition (Kriegl et al., 2004), climate (Kobliansky et al., 2000; Pearson, 2000), exercise levels (Bass et al., 2002; Duncan et al., 2002), and a host of other environmental influences. Despite the importance of epigenetic factors, genetic factors are still considered to be the most important influence on bone formation. Slemenda et al (1996) investigated genetic effects 
on femoral neck geometry and bone mineral density using a twin study, where the heritability of bone mineral density, centre of mass and resistance to forces of the femoral neck were estimated as $0.72,0.70$, and 0.94 respectively. This quantity can be calculated by graphing the fitness of the offspring versus that of their parents, and measuring the slope of the line. A sample graph used to calculate the heritability is shown in Figure 14. The 10\% loading moment variation included in our model gives rise to a heritability of approximately 0.68 , which means that roughly $68 \%$ of the phenotypic characteristics will be attributable to genetic influences, while the other $32 \%$ of effects observable in the phenotype are attributable to the external environment. Therefore, the heritability of the genes in our model of $68 \%$ corresponds well to the experimentally calculated values for the heritability of the bone mass density and centre of mass of the femoral neck as detailed above and it provides a corroboration of our model.

The simulation has elements in common with that of Siegal and Bergman (2002), who model an evolving developmental-genetic system. Their model suggests that canalization may occur as a result of developmental processes even without the influence of stabilizing selection. Canalization, as described by Waddington (1975) is the buffering of animal development such that, in the face of genetic and environmental perturbations, the optimum end result is still produced. In the results presented, none of the populations converge to the optimum mechanoregulatory response that would grow the fittest structure; that is to say they do not converge to $c=1.29$. This outcome seems to contradict the well known optimality assumption, which is stated by Emlen (1987) as "the phenotype(s) characterizing the best adapted individuals possible in a population comes to dominate that population". Lewontin (1987) suggests that historical accidents of environmental fluctuation and genetic drift are responsible for shaping a species, rather than optimality. Our simulations would seem to support the view that optimization never takes control over the random elements and therefore that optimum structures are not evolved. Bagge (2000, citing Roesler, 1987) states that, although bone has often been considered an optimal structure under the given loading conditions, bone cannot immediately attain an 
optimum structure under a varying load, because of the slow speed of adaptation. However, Bagge (2000) does assume that bone adaptation is an optimization process, and that the bone seeks to reach an optimal internal structure, but this is not what we have found with our model. Instead, we have found that, due to combinatorial aspect of the genetic processes acting during evolution, the algorithms governing bone growth and development would not optimise the skeleton - instead non-optimal structures are formed.

Archaeological data can give useful information on the adaptation of bones. Ruff and Hayes (1983) demonstrate that modern femoral cross-sections are more cylindrical in shape than samples from the Pecos Pueblo femora (dating from between 1300-1650 A.D.), and suggest that this difference could be due to changes in loads, although they do admit that this is a tentative hypothesis. Could the genes governing bone formation and remodelling have shifted, even in the last 500 years, to influence this change in shape? Examination of fossils has shown that there is a difference in the rates of bone remodelling between Pleistocene groups (1.8 million to 11,000 years ago) and the Pecos Pueblo population (Abbott et al., 1996). The study showed that the later Pleistocene members of the genus Homo had greater bone mass, less bone turnover and smaller osteons than recent population, and this implies that bone remodelling laws have not remained constant over time, but are subject to selection pressures and consequently evolve in the same way as the skeletal structures themselves.

We conclude that the variation of mechanoregulation response within a population is dependant on the random evolutionary processes contained in the model, such as epigenetic variation and recombination. By exploring the mechanoregulation algorithms within a population, we would hope to better understand the remodelling response of the individual and its relationship to their genes, so as to better predict the effects of mechanical stimulation on bone growth and development, and in clinical procedures. Although limited by the simplicity of computational models relative to the complexity of skeletal growth, the model, we believe, does 
demonstrate that evolution with a finite number of genes can cause mechanoregulation to be trapped in far from equilibrium optimal states (Weinans and Prendergast, 1996).

In conclusion, we have simulated the evolutionary change in ontogenetic mechanoregulation. We predict that changes in mechanoregulation response occur, which indicates that is it possible that variation can exist among the parameters governing the mechanoregulation of bone growth, remodelling, and adaptation in extant populations. This result would suggest that non-deterministic equations should be used when modelling bone growth or remodelling, such as in the patient-specific analysis of skeletal responses due to bone disease or surgery. However, perhaps the most significant result is that convergence to the optimum mechanoregulation equation was not found and this implies that sub-optimal bone phenotypes always occurred in the population.

\section{Acknowledgments}

This research was funded by the Irish Research Council for Science, Engineering and Technology (IRCSET), under the National Development Plan (NDP). The authors are grateful to Professor Daniel Bradley (Department of Genetics, Trinity College Dublin), Professor Pádraig Cunningham, (Department of Computer Science, Trinity College Dublin), Professor Marjolein van der Meulen (Cornell University) and Professor Charles Turner (University of Indiana) for their advice on this research.

\section{References}

Alexander, R.M., 1981. Optima for Animals, Princeton University Press, Princeton, New Jersey.

Abbott, S., Trinkaus, E., Burr, D.B., 1996. Dynamic bone remodelling in later Pleistocene fossil hominids. Am. J. Phys. Anthrop. 99 (4), 585-601.

Bagge, M., 2000. A model of bone adaptation as an optimization process. J. Biomech. 33 (11), 1349-1357., doi:10.1016/S0021-9290(00)00124-X. 
Bass, S.L., Saxon, L., Daly, R.M., Turner, C.H., Robling, A.G., Seeman, E., Stuckey, S., 2002. The effect of mechanical loading on the size and shape of bone in pre-, peri-, and postpubertal girls: A study in tennis players. J. Bone Miner. Res. 17 (12), 2274-2280.

Boyce, B.F., Hughes, D.E., Wright, K.R., Xing, L.P., Dai, A.H., 1999. Recent advances in bone biology provide insight into the pathogenesis of bone diseases. Lab. Invest. 79 (2), 83-94.

Carter, D.R. ,Beaupré, G.S., 2001. Skeletal Form and Function, Cambridge University Press, MA.

Carter, D.R., Fyhrie, D.P., Whalen, R.T., 1987. Trabecular bone density and loading history: regulation of connective tissue biology by mechanical energy. J. Biomech. 20 (8), 785794., doi:10.1016/8756-3282(96)00143-3

Carter, D.R., Mikic, B., Padian, K., 1998. Epigenetic mechanical factors in the evolution of long bone epihyses. Zool. J. Linn. Soc. 123 (2), 163-178., doi:10.1006/zjls.1997.0108.

Cowin, S.C., Hegedus, D.H., 1976. Bone remodelling I: theory of adaptive elasticity. J. Elasticity 6, 313-326.

Currey, J.D., Alexander, R.M., 1985. The thickness of the walls of tubular bones. J. Zool. Lond. (A) 206 (Aug), 453-468.

de Jong, G., Gavrilets, S., 2000. Maintenance of genetic variation in phenotypic plasticity: the role of environmental variation. Genet. Res. 76 (3), 295-304.

de Vernejoul, M.C., Benichou, O., 2001. Human osteopetrosis and other sclerosing disorders: recent genetic developments. Calc. Tissue Int. 69 (1), 1-6.

Deng, C.X., Wynshaw Boris, A., Zhou, F., Kuo, A., Leder, P., 1996. Fibroblast growth factor receptor 3 is a negative regulator of bone growth. Cell 84 (6), 911-921., doi:10.1016/S0092-8674(00)81069-7.

Duncan, C.S., Blimkie, C.J.R., Kemp A., Higgs, W., Cowell, C.T., Woodhead, H., Briody, J.N., Howman-Giles, R., 2002. Mid-femur geometry and biomechanical properties in 15-to 18yr-old female athletes. Med. Sci. Sports Exerc. 34 (4), 673-681.

Emlen, J.M., 1987. The Shape of Optimality, in: Dupré, J. (Ed.), The Latest on the Best, MIT Press, MA, pp. 163-177.

Gilbert, S. F., 2000. Developmental Biology, 6 ed., Sinauer Associates, Inc., MA.

Hartl, D.L., Clark, A.G., 1997. Principles of Population Genetics, 3 ed., Sinauer Associates, Inc., MA.

Holland, J. H., 1975. Adaptation in Natural and Artificial Systems: An Introductory Analysis with Applications to Biology, Control and Artificial Intelligence, MIT Press, MA. 
Huiskes R., Ruimerman R., van Lenthe G.H., Janssen J.D., 2000. Effects of mechanical forces on maintenance and adaptation of form in trabecular bone. Nature 405 (6787), 704-706.

Huiskes, R., Weinans, H., Grootenboer, H.J., Dalstra, M., Fudala, B., Schooff, T.J., 1987. Adaptive bone remodelling applied to prosthetic design analysis. J. Biomech.,. 20 (11-12), 1135-1150., doi:10.1016/0021-9290(87)90030-3.

Karasik, D., Myers, R.H., Cupples, L.A., Hannan, M.T., Gagnon, D.R., Herbert, A., Kiel, D.P., 2002. Genome screen for quantitative trait loci contributing to normal variation in bone mineral density: The Framingham study. J. Bone Miner. Res. 17 (9), 1718-1727.

Klein, R.F., Turner, R.J., Skinner, L.D., Vartanian, K.A., Serang, M., Carlos, A.S., Shea, M., Belknap, J.K., Orwoll, E.S., 2002. Mapping quantitative trait loci that influence femoral cross-sectional area in mice. J. Bone Miner. Res. 17 (10), 1752-1760.

Kriegl, J.M., Oyserman, S., Roller, S.A., Blumenfeld, J., Volkman, S.K., Nashi, S., Hall, J.M., McCreadie, B.R., Goldstein, S.A., 2004. Influences of nutrition and physical forces on bone structure/function properties. Proceedings of the Orthopaedic Research Society.

Lanske B., Karaplis A.C., Lee K., Luz A., Vortkamp A., Pirro A., Karperien M., Defize L.H.K., Ho C., Mulligan R.C., AbouSamra A.B., Juppner H., Segre G.V., Kronenberg H.M., 1996. PTH/PTHrP receptor in early development and Indian hedgehog-regulated bone growth. Science 273 (5275), 663-666.

Lewontin, R.C., 1987. The Shape of Optimality, in: Dupré, J. (Ed.), The Latest on the Best, MIT Press, MA, pp. 151-159.

Long, F., Chung, U., Ohba, S., McMahon, J., Kronenberg, H.M., McMahon, A.P., 2004. Ihh signalling is directly required for the osteoblast lineage in the endochondral skeleton. Development 131 (6), 1309-1318., doi: 10.1242/dev.01006.

Marroig, G., Cheverud, J.M., 2004. Did natural selection or genetic drift produce the cranial diversification of neotropical monkeys? Am. Nat. 163 (3), E417-E428.

Martin, R.B., 2000. Towards a unifying theory of bone remodeling. Bone 26(1), 1-6., doi:10.1016/S8756-3282(99)00241-0.

McCammon, R.W. 1970. Human growth and development Thomas, Springfield, Ill., U.S.A.

Pauwels, F., 1941. Grundriß einer Biomechanik der Frakturheilung. In 34th Kongress der Deutschen Orthopädischen Gesellschaft. Ferdinand Enke Verlag, Stuttgart. (Biomechanics of the Locomotor Apparatus. Translated by Manquet, P., Furlong, R., (Eds.), 1980, Springer, Berlin, 375-407).

Peacock, M., Turner, C.H., Econs M.J., Foroud, T., 2002. Genetics of osteoperosis. Endocr. Rev. $23(3), 303-326$.

Pearson, O.M., 2000. Activity, climate and postcranial robusticity. Curr. Anthrop. 41 (4), 569607. 
Prendergast, P.J., 2002. Mechanics applied to skeletal ontogeny and phylogeny. Meccanica 37, 317-334.

Prendergast, P.J., Taylor, D., 1994. Prediction of bone adaptation using damage accumulation. J. Biomech. 27 (8), 1067-1076., doi:10.1016/0021-9290(94)90223-2.

Raup, D.M., 1986. Biological Extinction in Earth History, Science, 231, 1528-1533.

Roberts, J.A.F., Pembrey, M.E., 1985. An Introduction to Medical Genetics. $8^{\text {th }}$ ed., Oxford University Press.

Roesler, H., 1987. The history of some fundamental concepts in bone biomechanics. J. Biomech. 20 (11-12), 1025-1034., doi:10.1016/0021-9290(87)90020-0.

Ruff, C.B., Hayes, W.C., 1983. Cross-Sectional Geometry of Pecos Pueblo Femora and Tibiae- A Biomechanical Investigation: I. Method and General Patterns of Variation. Am. J. Phys. Anthrop. 60, 359-381.

Ruff, C.B., Larsen, C.S., Hayes, W.H., 1984. Structural changes in the femur with the transition to agriculture on the Georgia coast. Am. J. Phys. Anthrop. 64, 125-136.

Siegal, M.L., Bergman, A., 2002. Waddington's canalization revisited: Developmental stability and evolution. PNAS 99 (16), 10528-10532.

Skalak, R., Dasgupta, G., Moss, M., Otten, E., Dullemeijer, P., Vilmann, H., 1982. Analytical description of growth. J. Theor. Biol. 94 (3), 555-577.

Slemenda, C.W., Turner, C.H., Peacock, M., Christian, J.C., Sorbel, J., Hui, S.L., Johnston, C.C., 1996. The genetics of proximal femur geometry, distribution of bone mass and bone mineral density. Osteoperosis Int. 6 (2), 178-182.

Stevens, S.S., Beaupré, G.S., Carter, D.R., 1999. Computer model of endochondral growth and ossification in long bones: Biological and mechanobiological influences. J. Orthopaedic Res. 17 (5), 646-653.

Thompson, D.W., 1917. On Growth and Form. Cambridge University Press, Cambridge.

Tufto, J., 2000. Quantitative genetic models for the balance between migration and stabilizing selection. Genet. Res. 76 (3), 285-293.

Turner, C.H., Schriefer, J.L., Horton, L.G., Shultz, K.L., Beamer, W.G., 2004. Fine mapping of genetic trait loci linked to skeletal structure in mice. Proceedings of the Orthopaedic Research Society.

van der Meulen, M.C.H., Huiskes, R., 2002. Why Mechanobiology? A Survey Article, J. Biomech. 35 (4), 401-414., doi:10.1016/S0021-9290(01)00184-1.

van der Meulen, M.C.H., Beaupré, G.S., Carter, D.R., 1993. Mechanobiologic influences in long bone cross-sectional growth. Bone 14 (4), 635-642., doi:10.1016/8756-3282(93)90085-O. 
Waddington, C.H., 1975. The Evolution of an Evolutionist, Edinburgh University Press.

Waxman, D., Peck, J.R. 2003. The anomalous effects of biased mutation. Genetics 164 (4), 16151626.

Weinans H., Prendergast P.J., 1996. Tissue adaptation as a dynamical process far from equilibrium. Bone 19 (2), 143-149.

Wolff, J., 1986. The Law of Bone Remodelling. (Das Gesetz der Transformation der Knochen, Kirschwald, 1892). Translated by Maquet, P., Furlong, R. Springer, Berlin. 


\section{Figure Headers}

Figure 1: Diploid gene set. Each number is assigned from the 50 numbers constituting the gene pool.

Figure 2: Recombination

Figure 3: Torsional moment applied to simulation over lifetime of bone with 10\% Variation

Figure 4: Schematic of Computational Implementation

Figure 5: Effect of varying c on the growth of a bone. The bones shown were grown with the same mean loading curve.

Figure 6: Stress/Mass, or fitness values, for bones grown with varying values of $c$

Figure 7: Effect of 10\% variation in applied torsional moment on Stress/Mass

Figure 8(a): Gene values for population after 1000 generations (basic simulation, Gene-pool 1, Run A)

Figure 8(b): c Values for population after 1000 generations (basic simulation, Gene-pool 1, Run

A)

Figure 9(a): Gene values for population after 1000 generations (basic simulation, Gene-pool 2, Run A)

Figure 9(b): c Values for population after 1000 generations (basic simulation, Gene-pool 2, Run

A)

Figure 10(a): Gene values for population after 1000 generations (varying selection strength + mutations, Gene-pool 2, Run A)

Figure 10(b): c Values for population after 1000 generations (varying selection strength + mutations, Gene-pool 2, Run A)

Figure 11(a): Gene values for population after 1000 generations (varying selection strength + mutations, Gene-pool 2, Run B)

Figure 11(b): c Values for population after 1000 generations (varying selection strength + mutations, Gene-pool 2, Run B) 
Figure 12: Gene frequency data (varying selection strength + mutations, Gene-pool 2, Run A)

Figure 13: Gene frequency data (varying selection strength + mutations, Gene-pool 2, Run B)

Figure 14: Sample heritability graph (for one recombination). Heritability is calculated from slope of linear trend- line of average parental fitness vs. offspring fitness 


\section{Tables}

Table 1: Effect of varying strength of selection on probabilities of selection for a population of four individuals

Table 2: Test results of Gene-pools 1-4, including c value if population has converged. Within each Gene-pool, all of the four tests (Basic, + Mutations, + Varying Selection Strength, + Varying Selection Strength + Mutations) are performed with the same initial gene pool and initial population configuration. 


\begin{tabular}{lllll}
\hline Probability of Selection & Individual 1 & Individual 2 & Individual 3 & Individual 4 \\
\hline Selection Strength $=1$ & 0.1 & 0.2 & 0.3 & 0.4 \\
\hline Selection Strength $=0.5$ & 0.175 & 0.225 & 0.275 & 0.325 \\
\hline
\end{tabular}

Table 1: Effect of varying strength of selection on probabilities of selection for a population of four individuals

\begin{tabular}{|c|c|c|c|c|c|}
\hline Test & Run & Gene-pool 1 & Gene-pool 2 & Gene-pool 3 & Gene-pool 4 \\
\hline \multirow[t]{2}{*}{ Basic } & $\mathrm{A}$ & Non-convergence & $\begin{array}{l}\text { Convergence to } \\
\mathrm{c}=1.415\end{array}$ & $\begin{array}{l}\text { Convergence to } \\
\mathrm{c}=1.37\end{array}$ & $\begin{array}{l}\text { Convergence to } \\
\mathrm{c}=1.195\end{array}$ \\
\hline & B & Non-convergence & $\begin{array}{l}\text { Convergence to } \\
\mathrm{c}=1.39\end{array}$ & $\begin{array}{l}\text { Convergence to } \\
\mathrm{c}=1.365\end{array}$ & $\begin{array}{l}\text { Convergence to } \\
\mathrm{c}=1.22\end{array}$ \\
\hline \multirow[t]{2}{*}{ + Mutations } & A & $\begin{array}{l}\text { Convergence to } \\
\mathrm{c}=1.43\end{array}$ & $\begin{array}{l}\text { Convergence to } \\
\mathrm{c}=1.285\end{array}$ & $\begin{array}{l}\text { Convergence to } \\
\mathrm{c}=1.225\end{array}$ & $\begin{array}{l}\text { Convergence to } \\
\mathrm{c}=1.365\end{array}$ \\
\hline & B & $\begin{array}{l}\text { Convergence to } \\
c=1.37\end{array}$ & $\begin{array}{l}\text { Convergence to } \\
\mathrm{c}=1.105\end{array}$ & Non-convergence & $\begin{array}{l}\text { Convergence to } \\
\mathrm{c}=1.475\end{array}$ \\
\hline \multirow[t]{2}{*}{$\begin{array}{l}\text { + Varying Selection } \\
\text { Strength }\end{array}$} & A & $\begin{array}{l}\text { Convergence to } \\
\mathrm{c}=1.255\end{array}$ & $\begin{array}{l}\text { Convergence to } \\
\mathrm{c}=1.405\end{array}$ & $\begin{array}{l}\text { Convergence to } \\
\mathrm{c}=1.355\end{array}$ & Non-convergence \\
\hline & B & Non-convergence & Non-convergence & $\begin{array}{l}\text { Convergence to } \\
\mathrm{c}=1.305\end{array}$ & Non-convergence \\
\hline \multirow{2}{*}{$\begin{array}{l}\text { + Varying Selection } \\
\text { Strength }+ \\
\text { Mutations }\end{array}$} & A & Non-convergence & $\begin{array}{l}\text { Convergence to } \\
\mathrm{c}=1.115\end{array}$ & $\begin{array}{l}\text { Convergence to } \\
\mathrm{c}=1.47\end{array}$ & $\begin{array}{l}\text { Convergence to } \\
\mathrm{c}=1.335\end{array}$ \\
\hline & B & Non-convergence & Non-convergence & Non-convergence & $\begin{array}{l}\text { Convergence to } \\
\mathrm{c}=1.275\end{array}$ \\
\hline
\end{tabular}

Table 2: Test results of Gene-pools 1-4, including c value if population has converged. Within each Genepool, all of the four tests (Basic, + Mutations, + Varying Selection Strength, + Varying Selection Strength + Mutations) are performed with the same initial gene pool and initial population configuration. 


\begin{tabular}{l|l|l|l|l|l|}
\cline { 2 - 5 } $\mathbf{b}$ & $\mathbf{g}_{\mathbf{l a}}$ & $\mathbf{g}_{2}$ & $\mathbf{g}_{\mathbf{a}}$ & $\mathbf{g}_{4 \mathbf{a}}$ & $\mathbf{g}_{\mathbf{a}}$ \\
\cline { 2 - 5 } & $\mathbf{g}_{\mathbf{l b}}$ & $\mathbf{g}_{2 b}$ & $\mathbf{g}_{\mathbf{b}}$ & $\mathbf{g}_{4 \mathbf{b}}$ & $\mathbf{g}_{\mathbf{b}}$ \\
\hline
\end{tabular}

Figure 1: Diploid gene set. Each number is assigned from the 50 numbers constituting the gene pool. 


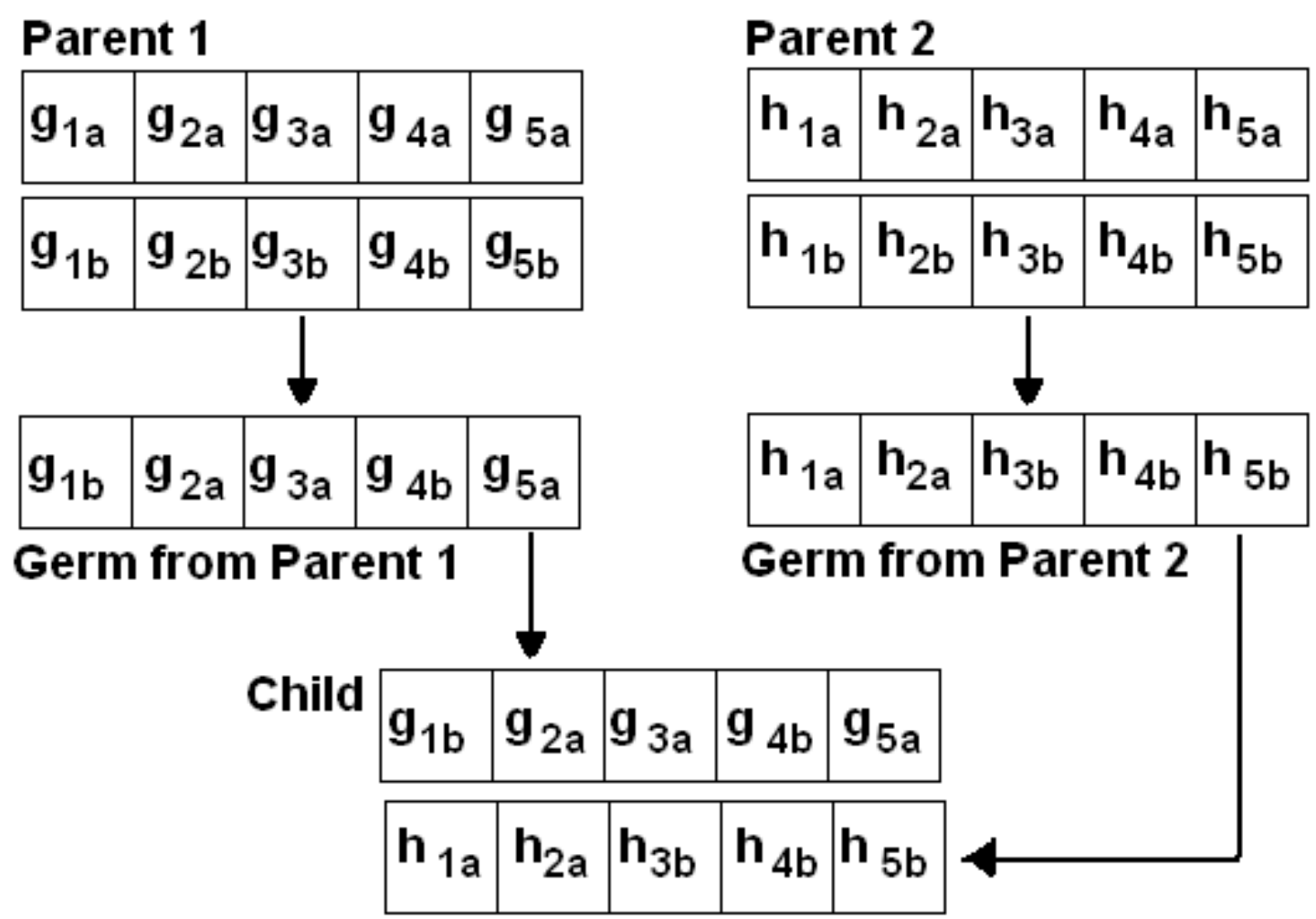

Figure 2: Recombination 


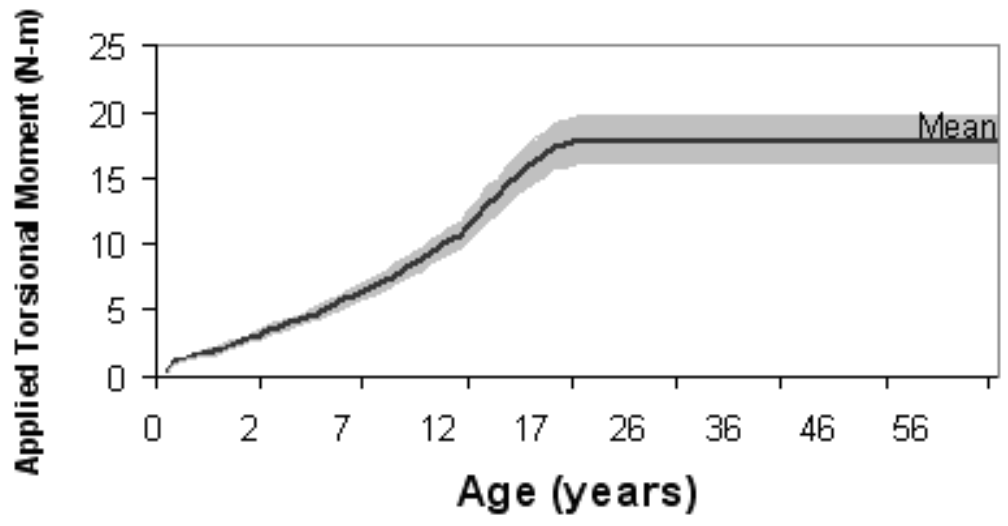

Figure 3: Torsional moment applied to simulation over lifetime of bone with 10\% Variation 


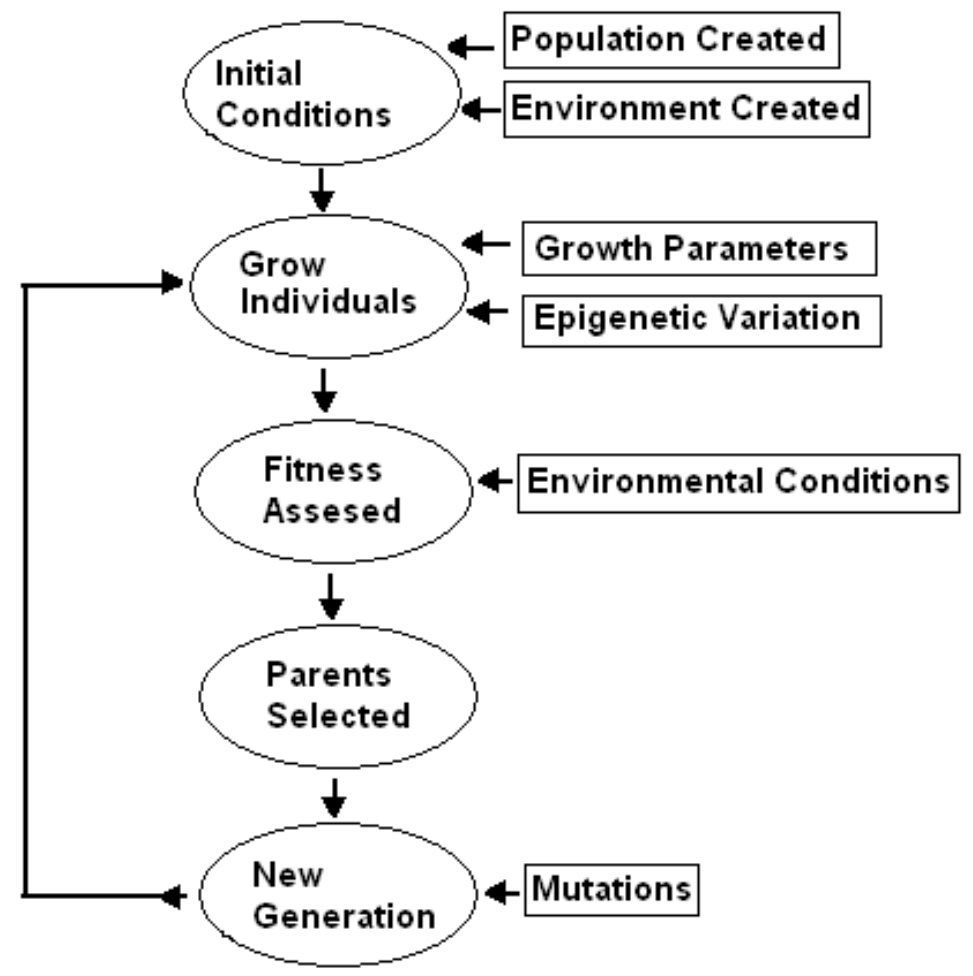

Figure 4: Schematic of Computational Implementation 


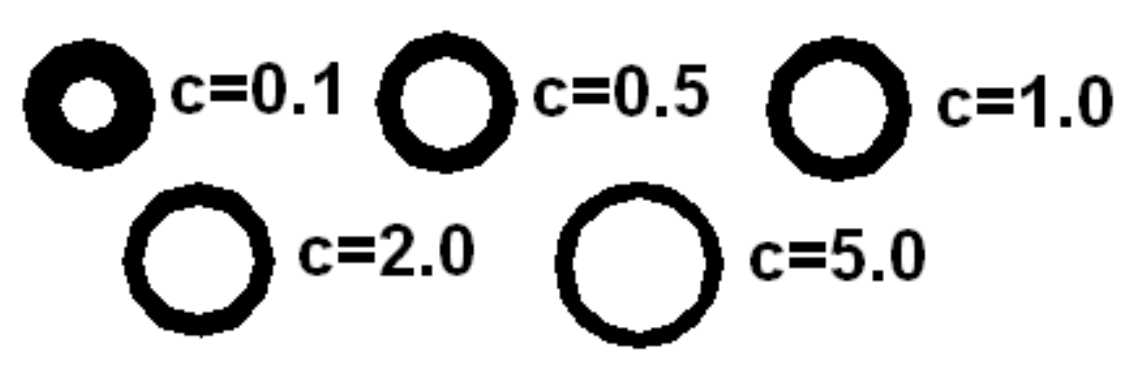

Figure 5: Effect of varying $c$ on the growth of a bone. The bones shown were grown with the same mean loading curve. 


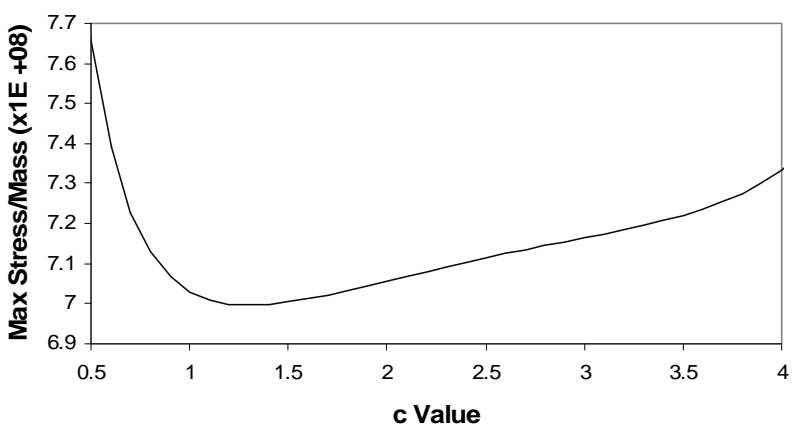

Figure 6: Stress/Mass, or fitness values, for bones grown with varying values of $c$ when mean torsional moment is applied, as shown in Fig.3 


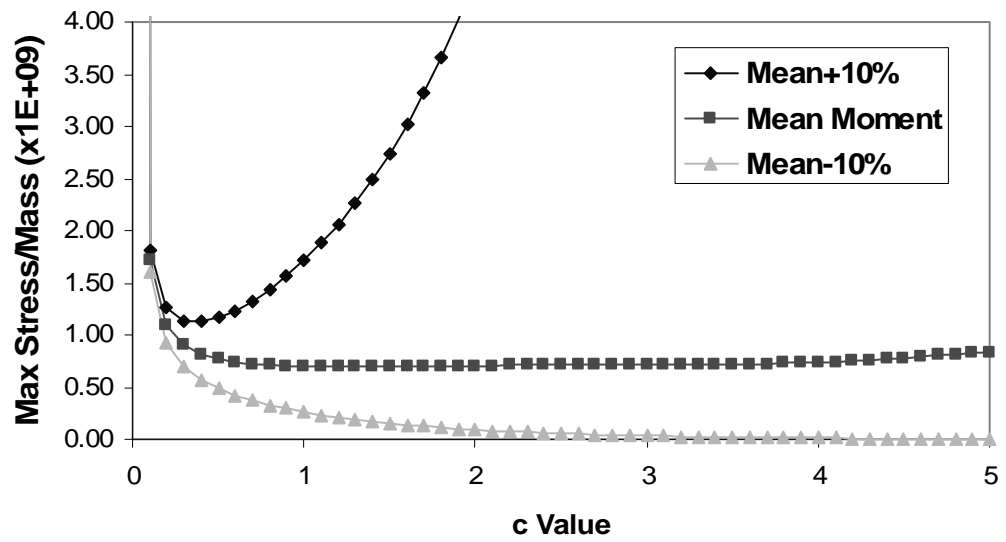

Figure 7: Stress/Mass, or fitness values, for bones grown with varying c, when the mean moment, the mean-10\% and the mean $+10 \%$ are applied. The middle curve is the same as the curve shown previously in Fig. 6. 
Basic Simulation (Gene-pool 1, Run A): Gene Values

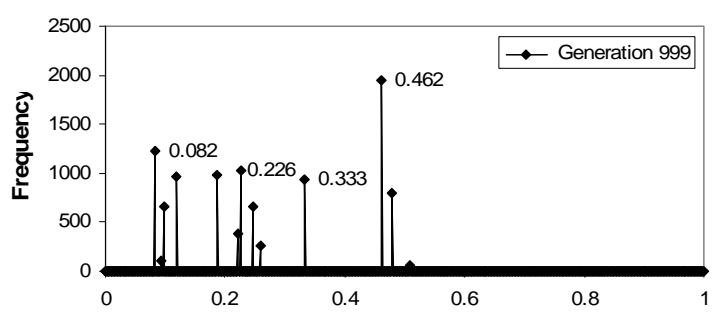

Figure 8(a): Gene values for population after 1000 generations (basic simulation, Gene-pool 1, Run A)
Basic Simulation (Gene-pool 1, Run A): c Values

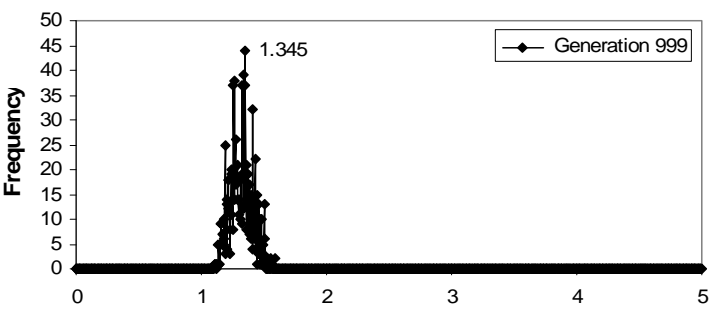

Figure 8(b): c Values for population after 1000 generations (basic simulation, Gene-pool 1, Run A) 
Basic Simulation (Gene-pool 2, Run A): Gene Values

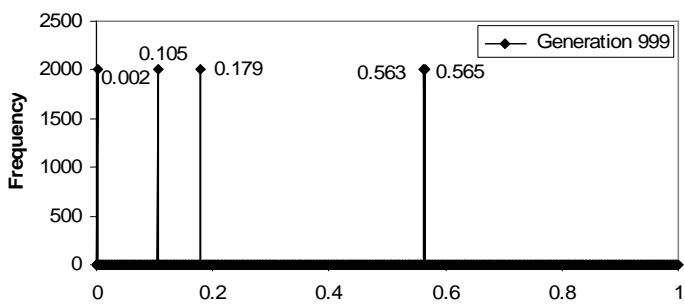

Figure 9(a): Gene values for population after 1000 generations (basic simulation, Gene-pool 2, Run A)
Basic Simulation (Gene Pool 2, Run A): c Values

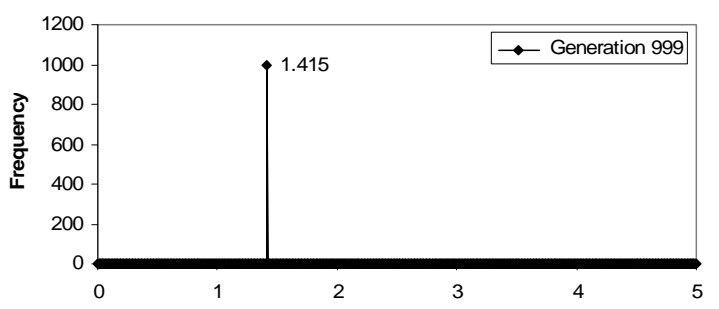

Figure 9(b): c Values for population after 1000 generations (basic simulation, Gene-pool 2, Run A) 
Varying Selection Strength + Mutations (Gene-pool 2, Run A): Gene Values

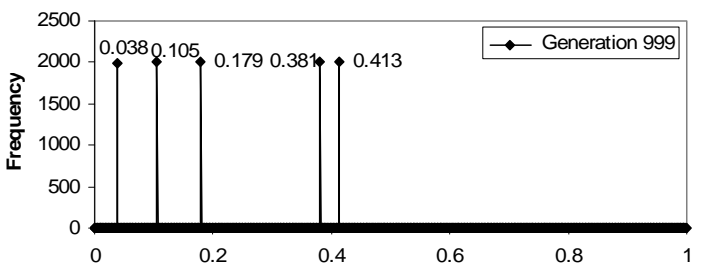

Figure 10(a): Gene values for population after 1000 generations (varying selection strength + mutations, Gene-pool 2, Run A)
Selection Slope + Mutations (Gene-pool 2, Run A):

c Values

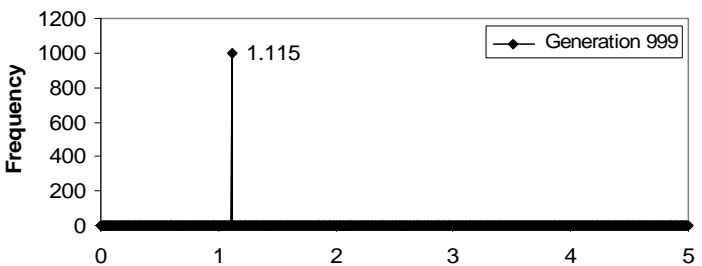

Figure 10(b): c Values for population after 1000 generations (varying selection strength + mutations, Gene-pool 2, Run A) 
Varying Selection Strength + Mutations (Gene-pool 2, Run B): Gene Values

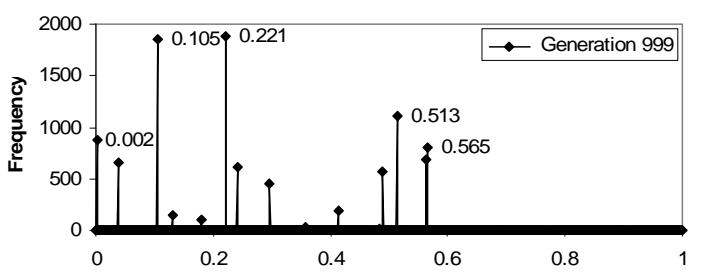

Figure 11(a): Gene values for population after 1000 generations (varying selection strength + mutations, Gene-pool 2, Run B)
Varying Selection Strength + Mutations

(Gene-pool 2, Run B): c Values

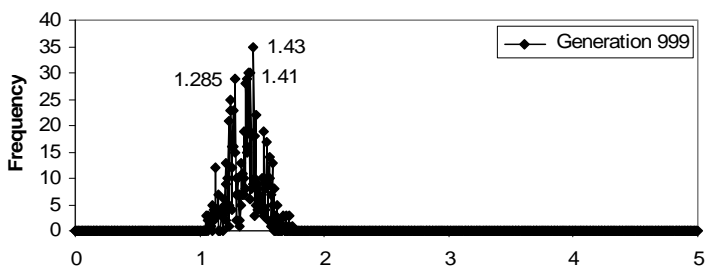

Figure 11(b): c Values for population after 1000 generations (varying selection strength + mutations, Gene-pool 2, Run B) 


\section{Varying Selection Strength + Mutations (Gene- pool 2, Run A): Gene Frequency Data}

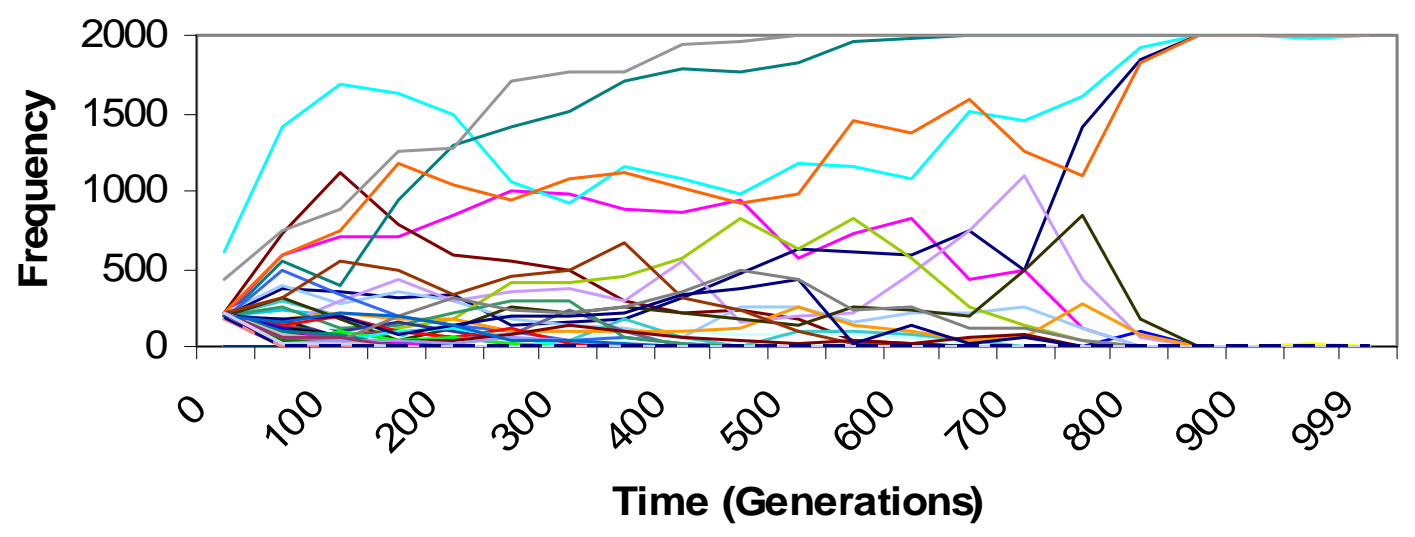

Figure 12: Gene frequency data (varying selection strength + mutations, Gene-pool 2, Run A) 


\section{Varying Selection Strength + Mutations (Gene- pool 2, Run B): Gene Frequency Data}

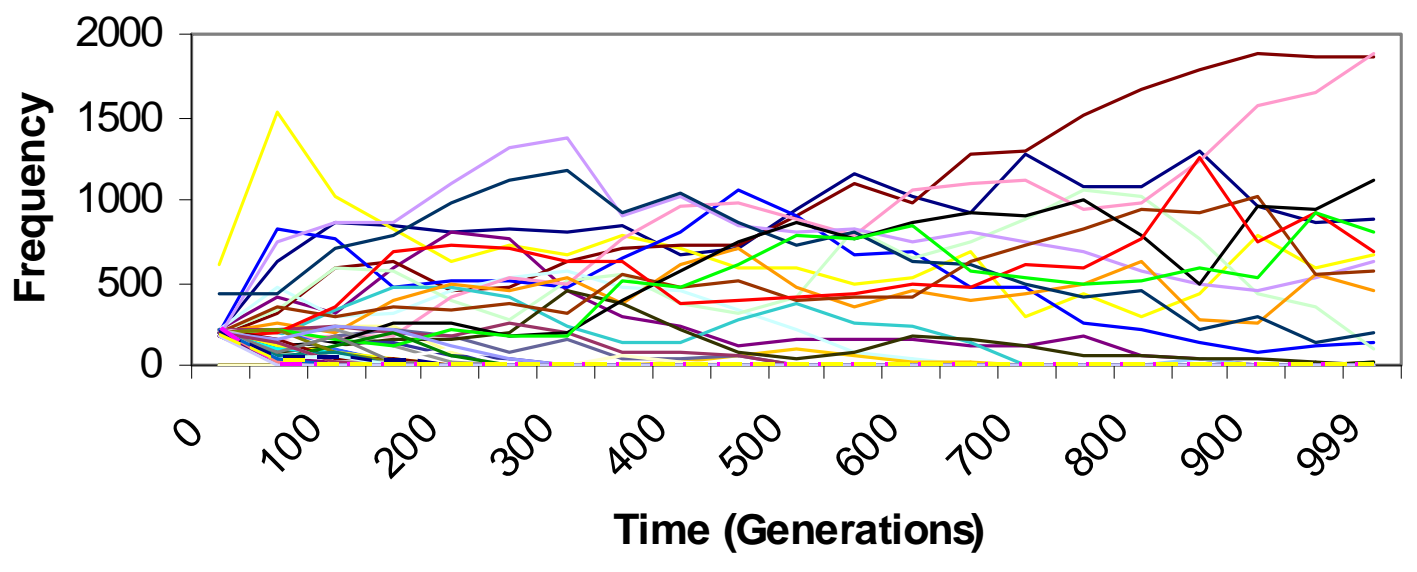

Figure 13: Gene frequency data (varying selection strength + mutations, Gene-pool 2, Run B) 


\section{Heritability Graph}

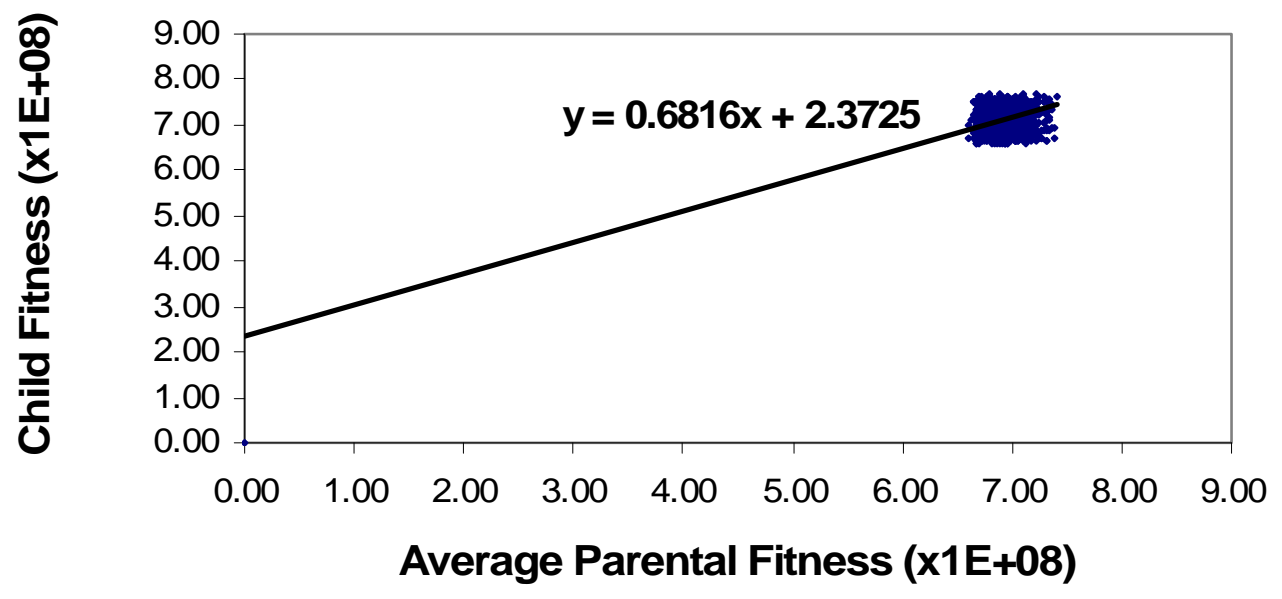

Figure 14: Sample heritability graph (for one recombination). Each point represents the fitness of the offspring versus the averaged fitness of the two parents for a sample generation. Heritability is calculated from the slope of the line through the points. 